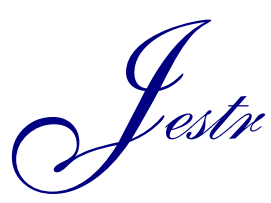

Research Article

\title{
Optimization Analysis of Improved Energy Detection based Cooperative Spectrum Sensing Network in Nakagami-m and Weibull Fading Channels
}

\author{
M. Ranjeeth ${ }^{1, *}$, Srinivas Nallagonda ${ }^{2}$ and S.Anuradha ${ }^{1}$ \\ ${ }^{I}$ Department of ECE, NIT-Warangal, Warangal, India, 506004 \\ ${ }^{2}$ Department of ECE M.L.R. Institute of Technology and Management Hyderabad, India, 500043
}

Received 7 December 2016; Accepted 10 April 2017

\begin{abstract}
In this paper, performance analysis for optimization of cooperative spectrum sensing (CSS) network in Nakagami-m and Weibull fading environments and comparison between them are presented. First, we derive the novel analytic expressions for probabilities of missed detection and false alarm for CSS network in both fading channels, assuming improved energy detector (IED) and selection combining (SC) diversity at each cognitive radios (CRs). Next, we optimize the network parameters such as number of CRs, energy detector arbitrary parameter, and normalized detection threshold at CR to obtain the optimal performance of CSS network. The impacts of several network parameters like: multiple antennas at each CR, number of CRs in CSS network, Nakagami and Weibull fading parameters, and sensing channel SNR on the performance of CSS network are investigated. The performance comparison between conventional and improved energy detectors has also been highlighted in this paper.
\end{abstract}

Keywords: Cooperative spectrum sensing, Fading, Improved energy detection, Total error rate, Optimization.

\section{Introduction}

Cognitive Radio (CR) concept is best technique for the efficient utilization of available radio spectrum. The $\mathrm{CR}$ users called secondary users (SUs) can utilize the available radio spectrum of the primary users (PUs) without interfering their operation [1]. Spectrum sensing is an important task to decide the vacant frequency bands (or spectrum holes) of the spectrum of the PUs. Conventional energy detection (CED) technique is one of the detection techniques which is used frequently to identify the existence of PU by calculating the energy of the received signal. The CED is the simplest one, non-coherent in nature, and also adds less complexity in a CR network [2]. The single CED based CR present in the network may face hidden terminal problem so that its performance is limited due to shadowing and fading effect exist in the nature. Thesedrawbacks can be eliminated by using multiple CRs are used in the network to sense the spectrum of a PU reliably is called as (cooperative spectrum sensing (CSS)) [3]. The CSS network gives better detection probability values though shadowing and fading effects are exist in the nature. The improved energy detection (IED) scheme is introduced to improve the detection probability and to overcome the limitations present in the CED scheme [4]. More precisely, the detection performance can be further improved significantly by replacing CED with IED at each CR in CSS network [5]. The IED measures the received signal amplitude (i.e. PU's

*E-mail address: ranjithmamidi2001@gmail

ISSN: $1791-2377$ @ 2017 Eastern Macedonia and Thrace Institute of Technology. All rights reserved. transmitted signal) with an arbitrary positive power (p).

In [6], an experimental approach of IED based spectrum sensing for CR is provided. Improved energy detection with data/decision fusion schemes are evaluated in [7]. The CSS network with IED scheme is considered and optimized values of network parameters are calculated using optimization technique to minimize the total error rate (It is the sum of probability of missed detection and false alarm) in [8]. It is necessarily required to optimize the parameters of the network to get optimal performance and to minimize the complexity of the network. In [9], optimized performance of CSS is achieved by optimizing the number of CR users, threshold value, and arbitrary power of the received signal in Rayleigh fading for single antenna at each CR user. In [10], multiple antennas at each CR with IED as detection scheme is used in Rayleigh fading channel. Optimization of CSS with CED in CR network is considered in [11]. The performance of a single CR user based on IED with multiple antennas in Rician and Hoyt fading channel is analyzed in [12]. However, optimal detection performance is not studied in [12]. Optimization of CSS network parameters with IED scheme is investigated in AWGN and Rayleigh fading channels [13]. The detection performance over $\dot{\eta}-\lambda-\mu$ fading distribution is investigated using CED scheme in [14].

It is an important to study optimal detection performance of IED based CSS network in Nakagami-m and Weibull fading channels because channels have been developed in modeling multi-path waves propagating in nonhomogeneous communication environments [15]-[17]. Weibull distribution is very flexible in both indoor and outdoor communication environments. Both Nakagami-m and Weibull distributions include as special cases the well- 
known Rayleigh and exponential distributions for a certain fading parameter values. In urban communication environments, both the distributions have the capability of accounting for propagation if the Rayleigh distribution fails e.g., digital enhanced cordless telecommunications (DECT) system. However, in spite of the usefulness of the distributions, the work related to CSS network with IEDs and multiple antennas over such fading conditions (Nakagami-m and Weibull) is not reported in the literature. This has motivated and the current work is dedicated to the analytical performance evaluation of CSS network with improved energy detection over Nakagami-m and Weibull fading channels. Finally, with this paper, our contributions to an existing literature are as follows:

- We derive a novel closed-form expression for missed detection probability $\left(P_{m}\right)$ with multiple antennas at each CR for Nakagami-m and Weibull fading in the S-channels.

- $\quad$ Selection combining (SC) diversity scheme is used at each CR to select the maximum value of the received signal at all the antennas. Imperfect R-channels having an error probability ( $\mathrm{r}$ ) are considered between CRs and FC.

- The closed-form expressions for optimal normalized detection threshold $\left(\lambda_{n, o p t}\right)$ and IED parameter $\left(p_{\text {opt }}\right)$ are presented for the case with $\mathrm{M}=1$ and $\mathrm{N}=1$ in Nakagami-m and Weibull fading channels. The parameter $\left(\mathrm{N}, \lambda_{n}\right.$, and $\left.\mathrm{p}\right)$ value at which the total error rate $\left(Q_{m}+Q_{f}\right)$ is minimum is called optimum parameter value.

- MATLAB based simulations are performed to obtain optimal values for higher values of $\mathrm{M}$ and N. A pair of probabilities of detection $\left(Q_{d}\right)$ and false alarm $\left(Q_{f}\right)$ at $\mathrm{FC}$ for higher values $\mathrm{N}$ and $\mathrm{M}$ are investigated. The optimum values for other network parameters also calculated.

- The impacts of error in the R-channel, fading parameters, multiple antennas at each $\mathrm{CR}$, and average S-channel SNR on the proposed CSS network are investigated. Comparison between CED and IED with and without diversity antenna cases is also evaluated.

The remaining sections are organized as follows: Section II describes the proposed CSS network model with suitable analytical frame work. Section III presents derivations for optimization of network parameters such as $N_{\text {opt }}, p_{\text {opt }}$, $\lambda_{n, \text { opt }}$. Simulation results are described in section IV. Finally, conclusions are given in section V.

\section{Proposed Network Model}

This section describes about proposed CSS network model and suitable analytical framework to evaluate the network performance. The main difference between conventional and improved energy detectors is, earlier one operates on squaring of the received signal and later one takes the arbitrary power (p) of the received signal. With the aid of this additional function the detection performance of a CR can be improved. Fig. 1 shows the proposed CSS network model with $\mathrm{N}$ number of CRs, a FC and a PU. Each CR consist of multiple receiving antennas (M) and each CR uses IED scheme. Both the FC and the PU use single antenna.
The channel present between PU and CR is called as sensing channel (S-channel), through which each $\mathrm{CR}$ senses the PU's activity (present or absent) with multiple antennas and stores the sensing information with in it for IED operation and takes local decision about the PU. We consider Nakagami-m or Weibull fading in the S-channels. The channel present between $\mathrm{CR}$ and FC is called as reporting channel (R-channel), through which each $\mathrm{CR}$ forwards its local decisions to the FC. We considered that an imperfect binary symmetric channel (BSC) in R-channel having an error probability (r). Next, the FC combines all the decisions received from all the CRs through $\mathrm{R}$-channel with ' $\mathrm{r}$ ' and makes the final decision about the PU using OR-logic at FC.

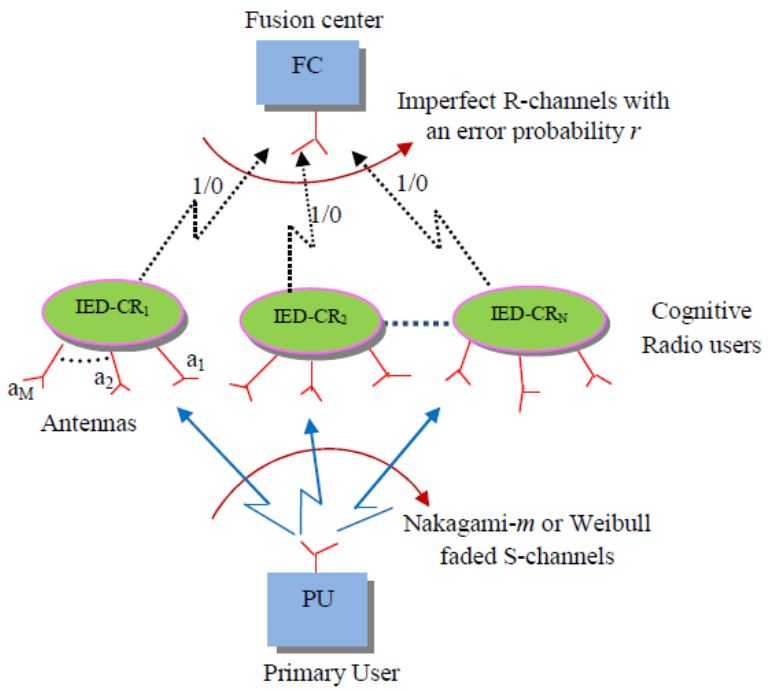

Fig.1 A proposed cooperative spectrum sensing (CSS) network model.

Each CR uses an IED scheme on the signals received by multiple antennas to get different signal strengths (test statistics) and uses selection combining (SC) diversity to select the maximum signal strength. Then, maximum test statistics at the output of SC is compared with a predetermined threshold $(\lambda)$ which is set at each CR to decide the existence of PU's activity. To decide the existence of PU's activity, two hypotheses, namely: $H_{0}$ (indicates PU's absence) and $H_{1}$ (indicates PU's presence) are assumed in the each $\mathrm{CR}$. The received signal at $\mathrm{i}$-th antenna $(\mathrm{i}=1 \ldots \mathrm{M})$ in each $\mathrm{CR}, y_{i}(\mathrm{t})$ can be written as:

$y_{i}(\mathrm{t})= \begin{cases}n_{i}(t) & : H_{0} \\ h_{i}^{*} s(t)+n_{i}(t) & : H_{1}\end{cases}$

where $\mathrm{s}(\mathrm{t})$ is the PU's signal with energy $E_{s}$ and $n_{i}(\mathrm{t})$ is the noise at $\mathrm{i}$-th antenna. Additive white Gaussian noise (AWGN) with normal distributed is assumed at each CR. Schannel fading coefficient for $\mathrm{i}$-th antenna is denoted as $h_{i}$. It is considered that each CR with multiple antenna contains the IED to detect the PU's activity. The expression for test statistics of i-th antenna after performing arbitrary power parameter $(p)$ on the received signal is [10]:

$$
W_{i}=y_{i}^{p} \quad p>0
$$

For $\mathrm{p}=2$, eq. (2) becomes the test statistics for the CED. If the $\mathrm{p}$ value is set to be more than 2 i.e., $\mathrm{p}>2$, eq. (2) becomes the test statistics for the IED. Selection combining (SC) diversity is operated over all the statistics of ' $w_{i}$ ' 
values, selects largest value denoted as $\mathrm{Z}$, and compared with predetermined detection threshold ' $\lambda$ ' to make decision about the PU [10]:

$$
Z>\lambda: H_{1} \& Z<\lambda: H_{0}
$$

where $\lambda$ can be obtained as $\lambda=\lambda_{n} \sigma_{n}^{p} . \quad \lambda_{n}$ is the fixed normalized detection threshold [9]-[10]. The probability density function (PDF) for Nakagami-m fading channel under hypothesis $H_{1}$ can be evaluated using [18] as:

$$
f_{y_{i} \mid \mathrm{H}_{1}}(x)=\frac{2 x^{\frac{2 m-1}{p}}}{p \Gamma(m)}\left(\frac{m}{\mathrm{E}_{\mathrm{s}} \sigma_{h}^{2}+\sigma_{n}^{2}}\right)^{\mathrm{m}} \exp \left(-\frac{m x^{2 / p}}{\mathrm{E}_{\mathrm{s}} \sigma_{h}^{2}+\sigma_{n}^{2}}\right)
$$

The ' $P_{m}$ ' expression for Nakagami-m fading channel $\left(P_{m, \text { Naka }}\right)$, can be obtained by using $(4)$ and (5):

The missed detection probability $\left(P_{m}\right)$ for any fading channel is calculated using:

$$
\begin{aligned}
& P_{m}=p r\left(\mathrm{y}<\lambda \mid \mathrm{H}_{1}\right)=\int_{0}^{\lambda} f_{w_{i} \mid \mathrm{H}_{1}}(\mathrm{y}) \mathrm{dy} \\
& =\int_{0}^{\lambda} \frac{2 x^{\frac{2 m-1}{p}}}{p \Gamma(m)}\left(\frac{m}{\mathrm{E}_{\mathrm{s}} \sigma_{h}^{2}+\sigma_{n}^{2}}\right)^{\mathrm{m}} \exp \left(-\frac{m x^{2 / p}}{\mathrm{E}_{\mathrm{s}} \sigma_{h}^{2}+\sigma_{n}^{2}}\right) d x \\
& =\frac{1}{\Gamma(m)} \int_{0}^{\frac{-m \lambda^{2} / p}{E_{s} \sigma_{h}^{2}+\sigma_{n}^{2}} \mathrm{t}^{m-1} \exp (-t) d t} \\
& =\frac{1}{\Gamma(m)} \gamma\left(m, \frac{m \lambda^{2 / p}}{E_{s} \sigma_{h}^{2}+\sigma_{n}^{2}}\right)^{M}
\end{aligned}
$$

where $\gamma($,$) is lower incomplete gamma function.$

Similarly, the PDF under $H_{1}$ for Weibull fading channel can be evaluated as:

$$
\begin{aligned}
& f_{w_{i} \mid \mathrm{H}_{1}}(x)=\frac{2 x^{(2 / p)-1} C}{p}\left[\frac{\Gamma(\mathrm{P})}{\left(E_{s} \sigma_{h}^{2}+\sigma_{n}^{2}\right)}\right] * \\
& *\left(x^{2 / p}\right)^{\mathrm{C}-1} \exp \left(-\left\{\frac{x^{2 / p} \Gamma(\mathrm{P})}{\left(E_{s} \sigma_{h}^{2}+\sigma_{n}^{2}\right)}\right\}^{C}\right.
\end{aligned}
$$

where $C=V / 2, P=1+1 / C$ and $\mathrm{V}$ - is Weibull fading parameter.

The ' $P_{m}$ ' expression for Nakagami-m fading channel $\left(P_{m, W e i}\right)$, can be obtained by using (7) and (5):

$$
\begin{aligned}
& =\int_{0}^{\lambda} \frac{2 x^{(2 / p)-1} C}{p}\left[\frac{\Gamma(\mathrm{P})}{\left(E_{s} \sigma_{h}^{2}+\sigma_{n}^{2}\right)}\right]\left(x^{2 / p}\right)^{\mathrm{C}-1} \exp \left(-\left\{\frac{x^{2 / p} \Gamma(\mathrm{P})}{\left(E_{s} \sigma_{h}^{2}+\sigma_{n}^{2}\right)}\right\}^{C}\right) d x \\
& P_{m}=\left[1-\exp \left(-\left\{\frac{\lambda^{2 / P} \Gamma(\mathrm{P})}{\sigma_{n}^{2}(1+\gamma)}\right\}\right)\right]^{M}
\end{aligned}
$$

The ' $P_{m}$ ' expression for Rayleigh fading channel $\left(P_{m, \text { Ray }}\right)$ can be obtained by substituting either $\mathrm{V}=2$ in eq. (8) or $\mathrm{m}=1$ in eq. (6) as [12]:

$$
P_{m}=\left[1-\exp \left(-\frac{\lambda^{2 / P}}{\sigma_{n}^{2}(1+\gamma)}\right)\right]^{M}
$$

where $\gamma=E_{s} \sigma_{h}^{2} / \sigma_{n}^{2}$ is average sensing channel SNR.

The above expression is exactly match with [10]. It is well known that ' $P_{f}$ ' expression under any fading channel (Rayleigh, Nakagami-m, and Weibull fading) is same as the ' $P_{f}$ ' expression for non-fading because ' $P_{f}$ ' is an independent of $\bar{\gamma}$. For non-fading case, ' $P_{f}$ ' can be derived using exponential distribution given in [9], [12] as:

$$
P_{m}=\left(1-\exp \left(-\lambda^{2 / p} / \sigma_{n}^{2}\right)\right)^{M}
$$

The R-channel is assumed to be BSC and an imperfect channel with an error probability rate $(r)$. Final values of false alarm $\left(Q_{f}\right)$ and missed detection $\left(Q_{m}\right)$ probabilities with all CRs in CSN network using OR-logic at FC having an error rate (r) can be expressed as following [9], [10]

$$
\begin{aligned}
& Q_{f}=1-\left[\left(1-P_{f}\right)(1-r)+r P_{f}\right]^{N} \\
& Q_{m}=\left[P_{m}(1-r)+r\left(1-P_{m}\right)\right]^{N}
\end{aligned}
$$

where $P_{m}$ and $P_{f}$ are the individual missed and false alarm probabilities at each CR. Finally, total error rate value $\mathrm{Z}(\mathrm{N})$ can be evaluated as:

$$
Z(N) \cong Q_{f}+Q_{m}
$$

\section{Optimization of Proposed Network Parameters}

To get the optimized performance of CSS network, network parameters such as $\mathrm{N}, \lambda_{n}$, and $\mathrm{p}$ are to be optimized. In this section, the expressions for optimum number of CRs ' $N$ ' is denoted as $\left(N_{\text {opt }}\right)$, optimum value of ' $\lambda_{n}$ ' is denoted as $\left(\lambda_{n, \text { opt }}\right)$, and finally optimum value of ' $\mathrm{p}$ ' is denoted as $\left(p_{\text {opt }}\right)$ are derived in the following subsequent subsections.

\subsection{Optimization of Number of CR users (N):}

If the CSS network consist of more number of CRs may experience large delay while making the decision about the PU. So, the large number has to be optimized to know how many optimum CRs are significantly utilized for making the final decision at FC. The optimum number (denoted as $N_{\text {opt }}$ ) of CRs can be calculated for a given values of average SNR, $\mathrm{p}$, and ' $\lambda_{n}$ ' by making $\Delta Z(N)$ equal to zero. Thus, $\Delta Z(N)$ can be calculated as: 


$$
\begin{aligned}
& \Delta Z(N)=Z(N+1)-Z(N)=0 \\
& \Rightarrow\left[\left(1-P_{f}\right)(1-r)+r P_{f}\right]^{N} \\
& +\left[P_{m}(1-r)+r\left(1-P_{m}\right)\right]^{N+1} \\
& -\left[\left(1-P_{f}\right)(1-r)+r P_{f}\right] \\
& \quad-\left[P_{m}(1-q)+q\left(1-P_{m}\right)\right]^{N}=0
\end{aligned}
$$

performing some algebraic analysis and using [11], an optimum ' $\mathrm{N}$ ' value can be obtained as

$$
N_{o p t} \cong\left\lceil\frac{\ln f_{2}\left(\mathrm{r}, P_{f}, P_{m}\right)}{\ln f_{1}\left(\mathrm{r}, P_{f}, P_{m}\right)}\right\rceil
$$

where $[$.$\rceil is the ceiling function. The functions f_{1}\left(\mathrm{r}, P_{f}, P_{m}\right)$ and $f_{2}\left(\mathrm{r}, P_{f}, P_{m}\right)$ can be expressed as

$$
\begin{gathered}
f_{1}\left(r, P_{f}, P_{m}\right)=\frac{P_{m}(1-r)+r\left(1-P_{m}\right)}{\left(1-P_{f}\right)(1-r)+r P_{f}} \\
f_{2}\left(r, P_{f}, P_{m}\right)=\frac{2 r P_{f}-r-P_{f}}{P_{m}-2 r P_{m}+r-1}
\end{gathered}
$$

where $P_{f}$ and $P_{m}$ are the pair of probabilities under any fading channel. Hence, $N_{\text {opt }}$ depends on type of fading, $\mathrm{p}$ and $\mathrm{r}$.

\subsection{Optimization of Normalized Threshold $\left(\lambda_{n}\right)$ :}

In order to estimate ' $\lambda_{n, o p t}$ ' value, differentiate eq. (14) with respect to ' $\lambda_{n}$ ' as:

$$
\begin{aligned}
\frac{\partial Z(\mathrm{n})}{\partial \lambda_{n}}= & -N(2 \mathrm{q}-1)\left[\left(1-P_{f}\right)(1-\mathrm{q})+\mathrm{qP}_{f}\right]^{N-1} \frac{\partial P_{f}}{\partial \lambda_{n}} \\
& +N(1-2 q)\left[P_{m}(1-q)+q\left(1-P_{m}\right)\right]^{N-1} \frac{\partial P_{m}}{\partial \lambda_{n}}
\end{aligned}
$$

where $\mathrm{q}=\mathrm{N}(1-2 \mathrm{r})$. For Nakagami-m fading channel and $\mathrm{M}=1, \partial P_{f} / \partial \lambda_{n}$ and $\partial P_{m} / \partial \lambda_{n}$ can be obtained as:

$$
\begin{aligned}
& \frac{\partial P_{f}}{\partial \lambda_{n}}=-\frac{2}{p} \lambda_{n}^{(2-\mathrm{p}) / p} \exp \left(-\lambda_{n}^{(2 / p)}\right) \\
& \frac{\partial P_{m}}{\partial \lambda_{n}}=\frac{2}{\Gamma(\mathrm{m}) p \lambda_{n}}\left(\frac{m \lambda_{n}^{2 / p}}{(1+\gamma)}\right)^{m} \exp \left(-\frac{m \lambda_{n}^{2 / p}}{1+\gamma}\right)
\end{aligned}
$$

The eq. (20) can be minimized by substituting $\mathrm{p}=2$ and $\mathrm{m}=2$ as

$$
\frac{\partial P_{m}}{\partial \lambda_{n}}=\frac{8 \lambda_{n}}{2(1+\gamma)^{2}} \exp \left(-\frac{2 \lambda_{n}}{(1+\gamma)}\right)
$$

Substitute (19) and (21) with $\mathrm{r}=0: 1, \mathrm{~N}=1$, and $\mathrm{M}=1$ in (18), then, we get a transcendental equation of the form:

$$
R-C_{2} \ln (\mathrm{R})-\mathrm{C}_{3}=0
$$

where

$$
\begin{aligned}
& C_{2}=\frac{(\mathrm{m}-1)(1+\gamma)}{m-(1+\gamma)} \\
& C_{3}=\frac{(1+\gamma) \ln \left(\frac{m^{m}}{\Gamma(\mathrm{m})(1+\gamma)^{m}}\right)}{m-(1+\gamma)}
\end{aligned}
$$

For $\mathrm{m}=2$, eq. (22) can be solved, and finally, $\lambda_{n, o p t, N a k}$ for various values of ' $\bar{\gamma}$ ' can be obtained as:

$\lambda_{n, o p t, N a k}=R^{p / 2}$

Similar procedure as stated above can be followed to obtain an expression for the optimal threshold for Weibull fading channel, $\lambda_{n, \text { opt,Wei }}$ with $\mathrm{r}=0: 1, \mathrm{M}=1$ and $\mathrm{N}=1$ as:

$\lambda_{n, o p t, W e i}=\left[\left\{\frac{C \ln (1+\bar{\gamma})}{\Gamma(P)\left[1-\left(\frac{1}{1+\bar{\gamma}}\right)^{C}\right]}\right\}\right]^{P / 2 C}$

An alternative expression for optimal threshold in Rayleigh fading channel, $\lambda_{n, o p t, \text { Ray }}$, can be obtained by substituting either $\mathrm{m}=1$ in eq. (25) or $\mathrm{C}=1$ in eq. (26) as:

$\lambda_{n, \mathrm{opt}}=\left[\frac{\ln (1+\gamma)}{(\gamma / 1+\gamma)}\right]^{p / 2}$

\section{Optimization of IED Parameter (p)}

In order to get $p_{\text {opt }}$ expression in both the fading channels, differentiate eq. (14) with respect to 'p' as:

$$
\begin{aligned}
\frac{\partial Z(\mathrm{n})}{\partial p}= & -N(2 r-1)\left[\left(1-P_{f}\right)(1-r)+r \mathrm{P}_{f}\right]^{N-1} \frac{\partial P_{f}}{\partial p} \\
& +N(1-2 r)\left[P_{m}(1-\mathrm{r})+r\left(1-P_{m}\right)\right]^{N-1} \frac{\partial P_{m}}{\partial p}
\end{aligned}
$$

For Rayleigh fading channel with $\mathrm{m}=1, p_{\text {opt,Ray }}$ can be obtained as

$p_{o p t}=\frac{2 \log \lambda}{\ln \left[\left(\frac{(1+\bar{\gamma}) \ln (1+\gamma) \sigma_{n}^{2}}{\bar{\gamma}}\right)\right]}$

For Weibull fading channel, expression for $p_{o p t, W e i}$ with $\mathrm{M}=\mathrm{N}=1$ can be obtained as 


$$
p_{\text {opt }}=\frac{2 \ln \lambda}{\ln \left\{\frac{C \ln (1+\gamma)}{\left[1-\left(\frac{1}{1+\gamma}\right)^{C}\right]}\left(\frac{1}{\Gamma(1+1 / C)}\right)\right\}^{1 / C}}
$$

The eq. (30) also provides an alternative expression for $p_{\text {opt }}$ for Rayleigh case for $\mathrm{C}=1$. For large values of $\mathrm{m}, \mathrm{M}$, and $\mathrm{N}$, solving the eqs. (18) and (28) becomes complex to get closed form of expressions for $p_{\text {opt }}$ and $\lambda_{n, o p t}$.

\section{Results and Discussions}

In the current section, the numerical results are presented and their discussions are provided. The effect of different values of the network parameters such as: $\left(\lambda_{n}\right),(\mathrm{p})$, the average S-channel SNR $(\bar{\gamma}),(\mathrm{M}),(\mathrm{N})$, and $(\mathrm{r})$ are also discussed. The proposed system is operated over the noisy, Nakagami-m and Weibull fading environments. The performance is evaluated using total error rate $\left(Q_{m}+Q_{f}\right)$ and probability of detection $\left(Q_{d}\right)$. Optimized values of network parameters such as $\left(N_{\text {opt }}\right),\left(\lambda_{n, o p t}\right)$, and $\left(p_{\text {opt }}\right)$ for the proposed system are investigated.

Fig. 2 shows the performance evaluation of a single CR user-based spectrum sensing as function of $\mathrm{p}$ for different values of $M$, namely $M=1$ and $M=3$. The performance is evaluated using $P_{m}$ and $P_{f}$ versus $\mathrm{p}$ in Nakagami-m and Weibull fading channels.

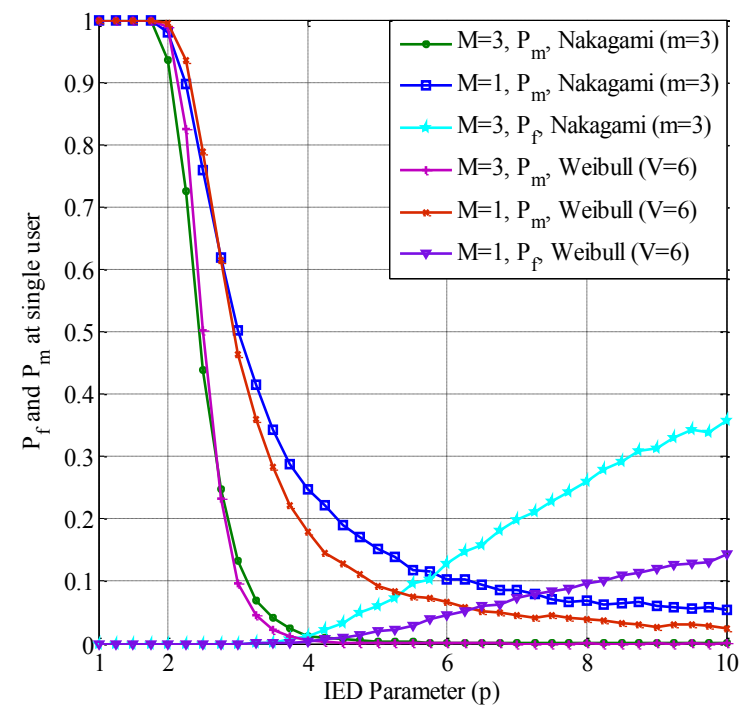

Fig. 2. The $P_{m}$ and $P_{f}$ in a single CR versus $\mathrm{p}$ under Nakagami-m $(\mathrm{m}=$ $3)$ and Weibull $(\mathrm{V}=6)$ fading channels for different values of $\mathrm{M}(\bar{\gamma}=10$ $\mathrm{dB}$ and $\left.\lambda_{n}=30\right)$.

In Fig. $2, P_{m}$ value decreases and $P_{f}$ value increases as $\mathrm{p}$ value increases. As we know that the CED operations would be obtained for $p=2$. The Pm is almost equal to 1 for $p=2$ while it reduces significantly for $p>2$ (i.e. CR with IED). It means that when CEDs are replaced with IEDs a significant improvement in the missed detection performance is obtained. It is also seen that for $\mathrm{p}=3: 25$, as ' $\mathrm{M}$ ' increases from 1 to $3, P_{m}$ value reduces from 0.4156 to 0.0695 under Nakagami-m fading channel and, it decreases from 0.3606 to 0.0467 under Weibull fading channel. This is because of the utilization of multiple number of antennas at each CR decreases the fading effect, hence the missed detection probability value decreases. The curve for $P_{f}$ versus $\mathrm{p}$ also shown under Nakagami-m and Weibull fading channels. It is seen that there is a trade-off between $P_{m}$ and $P_{f}$ with respect to parameter ' $p$ '. It can be concluded that higher and lower values of ' $p$ ' cannot be considered due to increases and decrease in the values of $P_{m}$ and $P_{f}$, respectively as 'p' increases i.e., optimum value of 'p' should be considered in order to maintain both $P_{m}$ and $P_{m}$ for a particular level. An optimum value is the value at which both $P_{m}$ and $P_{f}$ are minimum. In the following figures, the optimum values of $\mathrm{p}$, $\lambda_{n}$, and $\mathrm{N}$ for a cooperative CRs based network are investigated.

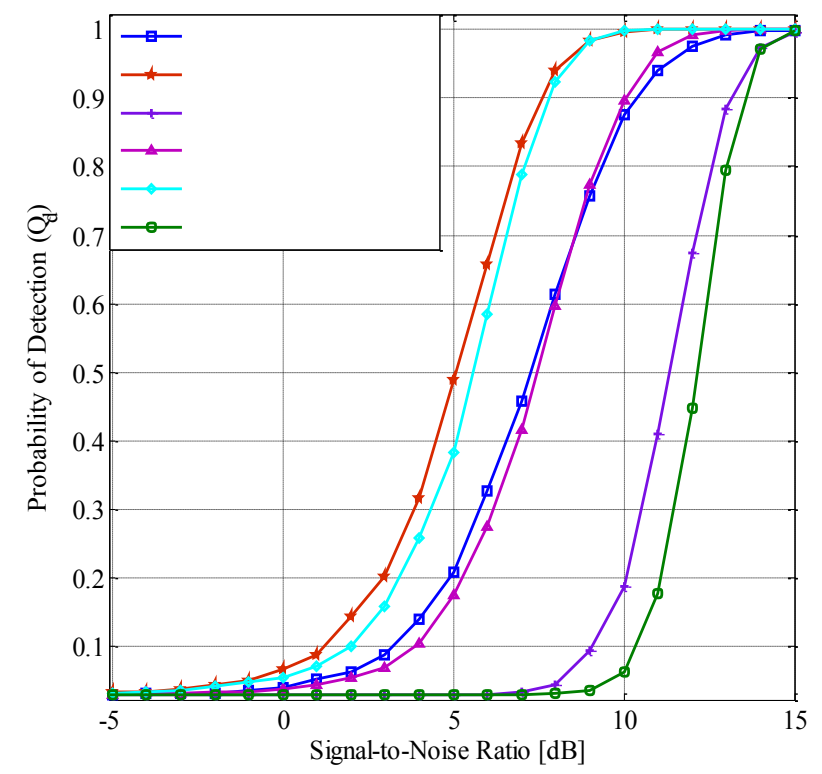

Fig. 3. $Q_{d}$ is shown as a function of $\bar{\gamma}$ for various values of $\mathrm{M}$ and $\mathrm{p}$ in Nakagami-m $(\mathrm{m}=3)$ and Weibull $(\mathrm{V}=6)$ fading channels $\left(\lambda_{n}=30, \mathrm{~N}\right.$ $=3$, and $\mathrm{r}=0.01)$.

Fig. 3, depicts Qd versus S-channel SNR $(\bar{\gamma})$ under Nakagami-m $(\mathrm{m}=3)$ and Weibull $(\mathrm{V}=6)$ fading channels for various values of $\mathrm{p}$ and $\mathrm{M}$ namely $\mathrm{p}=2, \mathrm{p}=3$ and $\mathrm{M}=$ $1, \mathrm{M}=3$. In Fig. 3 , as ' $\bar{\gamma}$ ' value increases, $Q_{d}$ value increases, this happens for any type of fading and for any values ' $\mathrm{p}$ ' and ' $\mathrm{M}$ '. This is due to fact that when ' $\bar{\gamma}$ ' value increases, noise effect decreases in the S-channel connected to each $\mathrm{CR}$ which leads to improved detection performance. It can be observed that for $\mathrm{M}=3$ and $\bar{\gamma}=6 \mathrm{~dB}$, as $\mathrm{p}$ increases from $p=2$ (i.e. CED operation is performed at each CR) to $p$ $=3$ (i.e. IED operation is performed at each CR), $Q_{d}$ increases from 0.03 to 0.7885 in Nakagami-m fading channel and from 0.04 to 0.835 in Weibull fading channel. This is due to increases in the received signal strength by power parameter ' $p$ '. Similarly, $Q_{d}$ value also increases as M value at each $\mathrm{CR}$ increases. This is due to increase in the diversity order of the antenna at each CR. For example, for $\mathrm{p}$ $=3$ and $\bar{\gamma}=6 \mathrm{~dB}$, as ' $\mathrm{M}$ ' increases from 1 to $3, Q_{d}$ increases from 0.2745 to 0.5854 in Nakagami-m and from 0.3266 to 0.6584 in Weibull fading channel. 


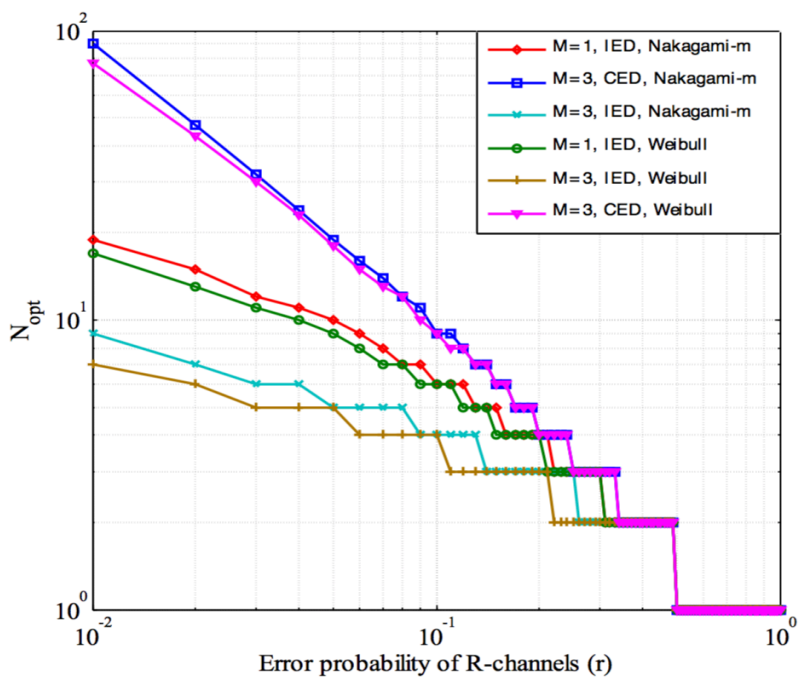

Fig. 4. The optimum number of CRs $\left(N_{o p t}\right)$ is shown as function of ' $r$ ' for various values of ' $M$ ' and for conventional and improved EDs ( $\mathrm{p}=2$ for CED, $\mathrm{p}=3$ for IED, $\lambda_{n}=20$ and $\bar{\gamma}=5 \mathrm{~dB}$ ).

It is necessary to find out $N_{\text {opt }}$ value which are exactly used to make final decision about the PU. In Fig. 4, $N_{o p t}$ is estimated in Nakagami-m $(\mathrm{m}=3)$ and Weibull $(\mathrm{V}=3)$ fading effects in S-channel for different error probabilities (r) in the R-channels. The $N_{\text {opt }}$ is also indicated for CSS network with CEDs and with IEDs. It can be seen from the graph that as ' $r$ ' value increases, $N_{\text {opt }}$ value decreases in both the fading channels. This is due to fact that when the higher values of ' $r$ ' are present in the R-channel, fusion center receives less number of binary decisions out of ' $N$ ' number of decisions. From the fig. we can also state that as $r$ value increases $N_{\text {opt }}$ value decreases due to increases in the arbitrary power parameter (i.e. p) of the received signal strength. Particularly, when $p$ increases from $p=2$ to $p=3$ (i.e. CEDs are replaced with IEDs) then $N_{\text {opt }}$ decreases from 30 to 3 in Weibull fading channel and it decreases from 32 to 6 in Nakagami-m fading channel. Finally, we can conclude that $N_{o p t}$ value is lesser for IEDs than that for CEDs to achieve the same performance level.

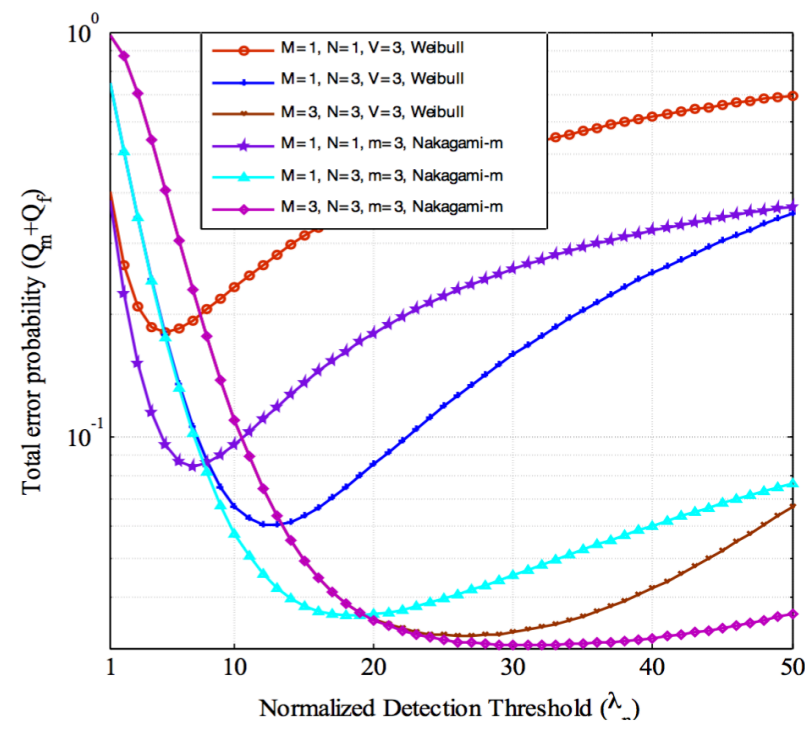

Fig. 5. Total error rate is shown as a function of $\lambda_{n}$, for various values of 'M' and ' $\mathrm{N}$ ' in Nakagami-m and Weibull fading channels $(\mathrm{p}=3, \bar{\gamma}=$ $10 \mathrm{~dB}$, and $\mathrm{r}=0.01)$.
In Fig. 5, an impact of $\lambda_{n}$ on $\left(Q_{m}+Q_{f}\right)$ is investigated in the presence of Nakagami-m $(\mathrm{m}=3)$ and Weibull $(\mathrm{V}=3)$ fading channels for various values of $\mathrm{N}$ and $\mathrm{M}$ namely $\mathrm{N}=$ $1, \mathrm{~N}=3$ and $\mathrm{M}=1, \mathrm{M}=3$ are used for this figure. It can be observed from Fig. 5 that $\left(Q_{m}+Q_{f}\right)$ value initially decreases with increasing the value of $\lambda_{n}$, later on it increases with further increasing in the value of $\lambda_{n}$. It can be observed that for a fixed values of $\mathrm{N}$ and $\mathrm{M}$, there exists an optimum $\lambda_{n}$ value is $\left(\lambda_{n, o p t}\right)$ at which $\left(Q_{m}+Q_{f}\right)$ value is minimum. The $\left(\lambda_{n, o p t}\right)$ value depends on $\mathrm{M}, \mathrm{N}$ and different fading environments. For a particular case, $\mathrm{N}=3$, Weibull fading channel, $\left(\lambda_{n, o p t}\right)$ value is 13 for $M=1$, while it is 26 for $\mathrm{M}=3$. Similarly, in Nakagami-m fading channel, $\left(\lambda_{n, \text { opt }}\right)$ values are 18 and 29 for $\mathrm{M}=1$ and $\mathrm{M}=3$ respectively for the same value of $\mathrm{N}$. It can be also be observed that the CSS network with SC diversity $(M>1)$ outperforms the CSS network without diversity $(M=1)$ under the same values simulation (network) parameters. When ' $\mathrm{M}$ ' value increases, $\left(P_{d}=1-P_{m}\right)$ increases at each CR due to diversity order increases which leads to decreases value of $\left(Q_{m}+Q_{f}\right)$ at each $\mathrm{CR}$ and further it decreases by cooperation of multiple CRs at FC. It is noted that the total error rate performance is worse for $\mathrm{N}=1$ (i.e. noncooperation) as compare to performance with $\mathrm{N}=3$ in both the environments. For example, for $\mathrm{M}=1$, as ' $\mathrm{N}$ ' increases from 1 to 3 total error rate decreases by $57.1 \%$ in Nakagami$\mathrm{m}$ fading channel and it decreases by $64.34 \%$ in Weibull fading channel. Similarly, for $N=3$, when ' $M$ ' increases from $M=1$ to $M=3$, total error rate decreases by $15.46 \%$ in Nakagami-m fading channel and it decreases by $49.29 \%$ in Weibull fading channel.

Fig. 6 shows an impact of $\mathrm{p}$ on the total error rate performance in Nakagami-m $\quad(\mathrm{m}=3)$ and Weibull $(\mathrm{V}=3)$ fading channels for different values of $(M=1),(M=3)$ and $(\mathrm{N}=1),(\mathrm{N}=3)$. As stated in Fig. 5, similar nature of the curve is observed in Fig. 6 also for various values of 'p', $\left(Q_{m}+Q_{f}\right)$ value decreases initially when the value of $\mathrm{p}$ increases and it increases with further increases in the value of ' $p$ '.

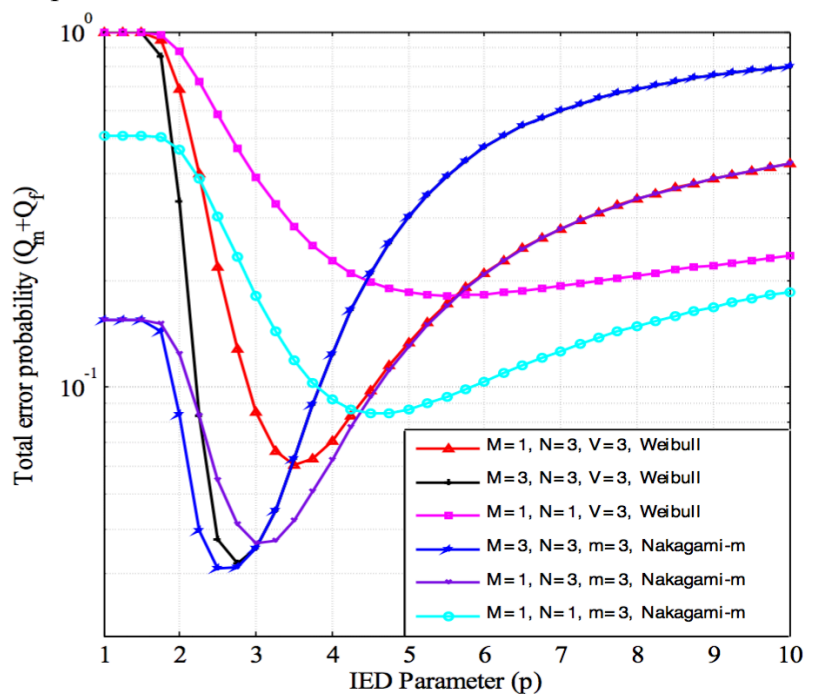

Fig. 6. Total error rate is shown as a function of $\mathrm{p}$ for various values of $\mathrm{M}$ and $\mathrm{N}$ in Nakagami-m and Weibull fading channels $\left(\lambda_{n}=20, \bar{\gamma}=10\right.$ $\mathrm{dB}$, and $\mathrm{r}=0.01$ ).

There exists an optimum $\mathrm{p}$ i.e., $p_{\text {opt }}$ value occurs at which $\left(Q_{m}+Q_{f}\right)$ is minimum. This is because of increasing in the value of ' $p$ ' makes decreasing in the value of detection threshold $\left(\lambda_{n}\right)$ and hence there is a chance to get the higher 
values of the probabilities false alarms at each $\mathrm{CR}$ and at FC. The optimal value of $\mathrm{p}\left(p_{\text {opt }}\right)$ depends on network parameters like: $\mathrm{M}, \mathrm{N}$ and different fading environments. Particularly, for $\mathrm{N}=\mathrm{M}=3, p_{\text {opt }}$ value is 2.5 with Nakagami-m and 2.75 with Weibull fading channel. Similarly, for $\mathrm{M}=\mathrm{N}=1, p_{\text {opt }}$ value is 4.5 with Nakagami-m and 5.5 with Weibull faded channel. We have observed that when $\mathrm{N}$ value increases form $\mathrm{N}=1$ to $\mathrm{N}=3$, there is a significant improvement in the total error rate performance. It can be concluded from figure that for a large number of $\mathrm{N}$, the optimum threshold shifts towards the origin. This means that $p_{\text {opt }}$ reduces as ' $\mathrm{N}$ ' increases but total error rate does not decrease at significant level. This is due to the fact that some CRs experience severe effect of noise in the Rchannels.

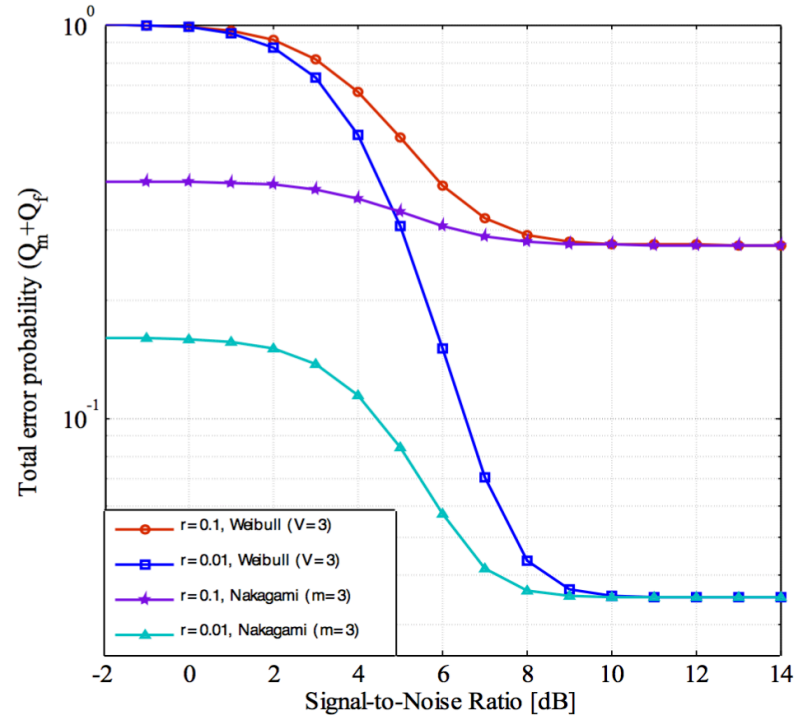

Fig. 7. Total error rate is shown as a function of $\bar{\gamma}$ for $r=0.1$ and 0.01 in Nakagami-m $(\mathrm{m}=3)$ and Weibull $(\mathrm{V}=3)$ fading channels $(\mathrm{M}=3, \mathrm{~N}=$ $3, \mathrm{p}=3$, and $\left.\lambda_{n}=30\right)$.

Fig. 7, is drawn between $\left(Q_{m}+Q_{f}\right)$ versus S-channel SNR $(\bar{\gamma})$ for different values of $r=0.1$ and $r=0.01$ in Nakagami-m and Weibull fading channels. We observe from Fig. 7 that as $\bar{\gamma}$ increases up to certain value (in $\mathrm{dB}$ ), total error rate decreases and latter it remains constant for further increases in the value of $\bar{\gamma}$. This type of behaviour is observed for both the fading channels and for any value of ' $r$ '. It can also be seen that the total error rate becomes constant at lower and higher values of $\bar{\gamma}$ for any value of ' $r$ '. For $\bar{\gamma}=6 \mathrm{~dB}$, as ' $\mathrm{r}$ ' value decreases from 0.1 to 0.01 , $\left(Q_{m}+Q_{f}\right)$ value decreases from 0.3915 to 0.1502 in Weibull fading channel and it decreases from 0.3074 to 0.0570 in Nakagami-m fading channel.

Table. I, Table II, and Table III describe the optimum values of $\mathrm{N}, \lambda_{n}$, and $\mathrm{p}$ respectively in Nakagami-m and Weibull fading channels for various network parameters. From Table.I, it can be noted that in both the fading channels $N_{\text {opt }}$ value decreases as ' $\mathrm{p}$ ' value increases while it increases with increases of ' $M$ ' value at $\bar{\gamma}=5 \mathrm{~dB}$. From Table. II and Table. III, it is observed that in both fading channels,

Table I. $N_{\text {opt }}$ for different channel and network parameters $\left(\lambda_{n}=20 \& \mathrm{r}=0.03\right)$

Fading factor

\begin{tabular}{c|c|c|c}
\hline $\mathrm{m}=3$ & 2 & 3 & 32 \\
$\mathrm{~m}=3$ & 3 & 1 & 12 \\
$\mathrm{~m}=3$ & 3 & 3 & 6 \\
$\mathrm{~V}=3$ & 2 & 3 & 30 \\
$\mathrm{~V}=3$ & 3 & 1 & 11 \\
$\mathrm{~V}=3$ & 3 & 3 & 5 \\
\hline
\end{tabular}

Table II $\lambda_{n, o p t}$ for different channel and network parameters $(\mathrm{p}=3 \& \mathrm{r}=0.01)$

\begin{tabular}{c|c|c|c}
\hline Fading factor & $\mathbf{M}$ & $\mathbf{N}$ & $\boldsymbol{\lambda}_{\boldsymbol{n}, \boldsymbol{o p t} \boldsymbol{t}}$ \\
\hline $\mathrm{m}=3$ & 1 & 1 & 31 \\
$\mathrm{~m}=3$ & 1 & 3 & 18 \\
$\mathrm{~m}=3$ & 3 & 3 & 7 \\
$\mathrm{~V}=3$ & 1 & 1 & 5 \\
$\mathrm{~V}=3$ & 1 & 3 & 13 \\
$\mathrm{~V}=3$ & 3 & 3 & 26 \\
$\mathrm{~V}=6$ & 1 & 1 & 7 \\
$\mathrm{~V}=6$ & 3 & 3 & 30 \\
\hline
\end{tabular}

Table III. $p_{\text {opt }}$ for different channel and network parameters $\left(\lambda_{n}=20 \& \mathrm{r}=0.01\right)$

\begin{tabular}{c|c|c|c}
\hline Fading factor & $\mathbf{M}$ & $\mathbf{N}$ & $\boldsymbol{p}_{\text {opt }}$ \\
\hline $\mathrm{m}=3$ & 1 & 1 & 4.5 \\
$\mathrm{~m}=3$ & 1 & 3 & 3.0 \\
$\mathrm{~m}=3$ & 3 & 3 & 2.5 \\
$\mathrm{~V}=3$ & 1 & 1 & 5.5 \\
$\mathrm{~V}=3$ & 1 & 3 & 3.5 \\
$\mathrm{~V}=3$ & 3 & 3 & 2.75 \\
$\mathrm{~V}=6$ & 1 & 1 & 4.5 \\
$\mathrm{~V}=6$ & 3 & 3 & 2.75 \\
\hline
\end{tabular}

$\lambda_{n, o p t}$ and $p_{\text {opt }}$ values are decreases when $\mathrm{M}$ and $\mathrm{N}$ values are increases at $\bar{\gamma}=10 \mathrm{~dB}$. The impact of fading parameters on $\lambda_{n, o p t}$ and $p_{\text {opt }}$ for a fixed values of $\mathrm{M}$ and $\mathrm{N}$ is also shown.

\section{Conclusion}

We have investigated how to choose an optimal number of CRs, optimal value of the normalized threshold, optimal value of the IED parameter in order to optimize the performance of a proposed CSS network. To obtain minimum total error rate, the parameters $\mathrm{N}, \lambda_{n}$, and $\mathrm{p}$ have been optimized. A novel analytical expressions for missed detection probability in both Nakagami-m and Weibull fading channels have been derived. It is shown that the derived expressions for optimal values of $\mathrm{N}, \lambda_{n}$, and $\mathrm{p}$ depend on the various network parameters. The impacts of parameter on the total error performance such as S-channel average SNR $(\bar{\gamma})$, multiple antennas $(\mathrm{M})$ at each CR, number of CRs $(\mathrm{N})$ and error probability in R-channel (r) have been investigated. A comparative study between CED and IED with and without diversity at each CR has also been studied. The application of this study is useful to design a optimized cooperative spectrum sensing network in the presence of various fading effects.

This is an Open Access article distributed under the terms of the Creative Commons Attribution Licence

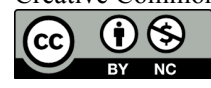




\section{References}

[1] J. Mitola and G. Maguire, "Cognitive radio: Making software radios more personal,’IEEE Personal Commun., vol. 6, no. 4, pp. 13-18, August 1999.

[2] F. F. Digham, M. -S. Alouini and M. K. Simon, "On the energy detection of unknown signals over fading channels," in Proceedings of IEEE International Conference on Communications (ICC'03), Alaska, USA, pp. 3575-3579, May 2003.

[3] G. Ganesan and Y. G. Li, "Cooperative spectrum sensing for cognitive radio networks," in Proceedings of IEEE Symp. New Frontiers in Dynamic Spectrum Access Networks (DySPAN'05), Baltimore, USA, pp.137-143, Nov. 2005.

[4] M. Lopez-Benitez and F. Casadevall, "Improved energy detection spectrum sensing for cognitive radio," IET Commun., vol. 6, issue. 8, pp.785-796, May 2012.

[5] Y. Chen, "Improved energy detector for random signals in Gaussian noise," IEEE Trans. Wireless. Comm., vol. 9, no. 2, pp. 558-563, Febrauary 2010.

[6] P. Sadhukhan, N. Kumar and M. R. Bhatnagar, "Improved energy detector based spectrum sensing for cognitive radio: an experimental study," in Proceedings of IEEE India Conference (INDICON'13)., Mumbai, India, pp. 1-5, December 2013.

[7] O. Olabiyi and A. Annamalai, "Improved energy detectors with data/decision fusion of partitioned time-domain samples," in Proceedings of IEEE International Conference on Connected Vehicles and Expo (ICCVE'13), Las Vegas, NV, pp.20-25, December 2013

[8] Shi-Qi Liu and Bin-Jie Hu, "Cooperative spectrum sensing based on improved energy detector for cognitive radios," in Proceedings of IEEE International conference on Signal Processing, Communications and Computing (ICSPCC'14), Guilin, pp. 1-4, August 2014.

[9] A. Singh, M. R. Bhatnagar and R. K. Mallik, "Optimization of cooperative spectrum sensing with an improved energy detector over imperfect reporting channel," in Proceedings of IEEE Vehicular Technology Conference (VTC Fall), September 211.

[10] A. Singh, M. R. Bhatnagar and R. K. Mallik, "Cooperative spectrum sensing in multiple antenna based cognitive radio network using an improved energy detector," in IEEE Communications Letters, vol. 16, no. 1, pp. 64-67, January 2012.

[11] W. Zhang, R. K. Mallik and K. B. Letaief, "Optimization of cooperative spectrum sensing with energy detection in cognitive radio networks," in IEEE Transactions on wireless communications, vol. 8, no. 12, pp. 5761-5766, December 2009.

[12] S. Nallagonda, A. Chandra, S. D. Roy and S. Kundu, "Performance of improved energy detector based cooperative spectrum sensing over Hoyt and Rician faded channels," IEICE communication express, vol. 2, No.7, pp. 319-324, July 2013.

[13] M. Ranjeeth, S. Behera, S. Nallagonda and S. Anuradha, "Optimization of cooperative spectrum sensing based on improved energy detector with selection diversity in AWGN and Rayleigh Fading," in Proceedings of International Conference on Electrical, Electronics, and Optimization Techniques (ICEEOT'2016), Chennai, India, pp. 1-5, March 2016.

[14] K. M. Captain and M. V. Joshi, "Energy detection based spectrum sensing over $\eta \dot{-} \lambda-\mu$ fading channel," in Proceedings of Eigth International Conference on Communication Systems and Networks (COMSNETS), Bangalore, pp.1-6, January 2016.

[15] Q. T. Vien, H. X. Nguyen and A. Nallanathan, "Cooperative spectrum sensing with secondary user selection for cognitive radio networks over Nakagami-m fading channels," IET communications, vol. 10, Iss. 1, pp. 91-97, 2016.

[16] M. Abdel-Hafez, K. Shuaib, O. El-B. Hamed,“ Analysis of spectrum utilization of cognitive radio networks in Nakagami fading channels," In proceedings of 82nd IEEE Vehicular Technology conference, Boston, USA, pp. 1-5, September 2015.

[17] P. C. Sofotasios, M. K. Fikadu, K. Ho-Van and M. Valkama, "Energy detection sensing of unknown signals over Weibull fading conditions," International Conference on Advanced Technologies for Communications (ATC-2013), pp. 414-419, 2013.

[18] M. K. Simon, M. S. Alouini, "Digital Communication over Fading Channels," John Wiley and Sons, 2nd edition, NJ, USA, December 2004. 